\title{
Idiopathische Lungenfibrose
}

\section{Langzeitbehandlung bremst die Progression}

- Für Patienten mit idiopathischer Lungenfibrose (IPF) gibt es derzeit keine Chancen auf Heilung. Vielmehr sind die Patienten auf eine Langzeittherapie angewiesen, die die Krankheitsprogression verlangsamen kann.

Aktuelle Langzeitdaten belegen jetzt, dass der niedermolekulare Tyrosinkinase-Inhibitor Nintedanib $\left(\mathrm{Ofev}^{\circledR}\right)$

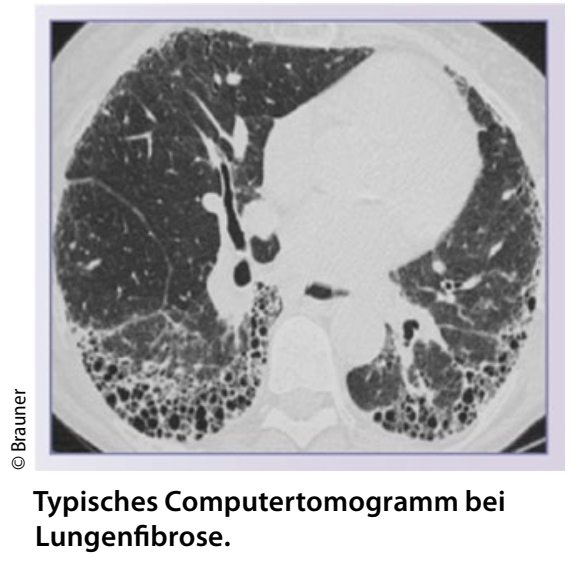

dauerhaft über zwei Behandlungsjahre die jährliche Abnahme der Lungenfunktion reduziert. Die Krankheitsprogression wird verlangsamt, berichtete Prof. Luca Richeldi, Southampton, Großbritannien.

Dies zeigt eine Zwischenauswertung der Langzeitstudie INPULSIS ${ }^{\circledR}$-ON, die beim Kongress der European Respiratory Society (ERS) erstmals präsentiert wurde [Crestani B et al. ERS 2015]. In den zulassungsrelevanten INPULSIS ${ }^{\circledR}$ Studien hat Nintedanib die jährliche Abnahme der forcierten Vitalkapazität (FVC) etwa halbiert [Cottin V et al. Eur Respir J. 2014; Behr J et al. Pneumologie. 2015; Costabel U et al. Eur Respir J. 2014]. In der unverblindeten Verlängerung blieb die Wirksamkeit über weitere 48 Wochen erhalten.

Die FVC-Abnahme betrug im ersten Behandlungsjahr durchschnittlich $89 \mathrm{ml}$ vs. $203 \mathrm{ml}$ unter Placebo (gepoolte Daten). Im zweiten Behandlungsjahr be- trug die mittlere FVC-Abnahme durchschnittlich $87 \mathrm{ml}$ bei allen Patienten, $96,4 \mathrm{ml}$ bei den Patienten unter fortgesetzter Therapie und 73,1 $\mathrm{ml}$ bei den $\mathrm{Pa}$ tienten, die die Therapie neu begannen.

Nintedanib ist auch geeignet für $\mathrm{Pa}$ tienten, die begleitend mit Antazida gegen gastroösophagealen Reflux oder mit Kortikosteroiden behandelt werden, wie Subgruppenanalysen der INPULSIS ${ }^{\circledR}$ Daten gezeigt haben [Raghu G et al. ERS 2015; Cottin V et al. ERS 2015].

Um die Progression der IPF wirksam $\mathrm{zu}$ bremsen, d.h. die fortschreitende Vernarbung des Lungengewebes zu verlangsamen, seien ein rechtzeitiger Therapiebeginn und eine hohe Therapietreue wichtig, betonte Richeldi.

Dagmar Jäger-Becker

- Symposium "A driving force in new treatments - latest insights from the Boehringer Ingelheim respiratory portfolio", ERS-Kongress; Amsterdam, September 2015 (Veranstalter: Boehringer Ingelheim)

\section{Therapie des Typ-2-Diabetes}

\section{Entscheidend ist das kardiovaskuläre Outcome}

_ SGLT2 sind Transportproteine, die über $90 \%$ der in den Nieren durch Filtration ausgeschiedenen Glukose wieder in die Blutbahn rückresorbieren. Empagliflozin (Jardiance ${ }^{\circledast}$ ) ist ein selektiver Inhibitor von SGLT2 und verbessert so die glykämische Kontrolle bei Typ-2-Diabetikern - und dies bei einem sehr niedrigen Hypoglykämierisiko. „Darüber hinaus beeinflusst die Substanz auch andere kardiovaskuläre Risikofaktoren günstig“, so Prof. Jochen Seufert, Freiburg. So kommt es zu einer Abnahme des Blutdrucks, des Gewichts, des viszeralen Fettgewebes und der Albuminurie.

\section{Verlängertes Überleben}

Für Empagliflozin konnte im Rahmen der EMPA-REG-OUTCOME ${ }^{\circledR}$-Studie und zwar erstmals für ein Antidiabeti- kum - eine günstige Wirkung im Hinblick auf die Verhinderung kardialer Ereignisse bei Typ-2-Diabetikern mit einem erhöhten kardiovaskulären Risiko nachgewiesen werden. Der kombinierte Endpunkt aus kardiovaskulärem Tod, nichttödlichem Herzinfarkt bzw. Schlaganfall wurde um 14\%, die kardiovaskuläre Mortalität um 38\%, die Gesamtmortalität um $32 \%$ und die Notwendigkeit für eine Krankenhausbehandlung wegen Herzinsuffizienz um 35\% gesenkt.

\section{Erstes Insulin-Biosimilar}

„In zahlreichen Studien konnte gezeigt werden, dass Insulin glargin im Vergleich mit einem NPH-Insulin (= Langzeit-, Intermediär- oder Basalinsulin) eine verlängerte Wirkdauer und ein niedrigeres Hypoglykämierisiko auf- weist", so Prof. Thomas Forst vom Profil Institut in Mainz. Im Rahmen der ORIGIN-Studie habe man auch die kardiovaskuläre Sicherheit dieses Insulins belegen können.

Mit Abasaglar ${ }^{\circledast}$ steht jetzt ein Insulin- glargin-Biosimilar zur Verfügung. Es weist eine identische Aminosäurensequenz wie das Original auf. „Aber auch das pharmakologische Profil und die klinische Wirkung sind durchaus vergleichbar, wie vorliegende Studiendaten zeigen“, so Forst. Dies gelte sowohl für die Senkung des $\mathrm{HbA}_{1 \mathrm{c}}$-Werts als auch für das niedrigere Hypoglykämierisiko.

\section{Dr. Peter Stiefelhagen}

- Satellitensymposium „Personalisierte Diabetestherapie: Effektivität und Sicherheit im Fokus", im Rahmen der DDGHerbsttagung, Düsseldorf, November 2015 (Veranstalter: Boehringer Ingelheim und Lilly) 